\title{
Alerta sobre a importância do conhecimento das radiações ionizantes e uso de protetores plumbíferos na radiologia odontológica
}

\author{
Alert on the importance of knowledge of ionizing radiations and the use of plumbiferous protectors \\ in dental radiology
}

Recebido: 04/10/2021 | Revisado: 10/10/2021 | Aceito: 13/10/2021 | Publicado: 14/10/2021

\author{
Marisa Macedo de Oliveira Costa \\ Universidade Brasil, Brasil \\ Email: rafa1996_@hotmail.com \\ Katia Reis do Nascimento Santos \\ Universidade Brasil, Brasil \\ E-mail: katiamillygaby6@gmail.com \\ Fabiana Molina de Oliveira \\ Universidade Brasil, Brasil \\ E-mail: fabyzinhamolina@hotmail.com \\ Denis Honorato Costa \\ Universidade Brasil, Brasil \\ E-mail: d_hto@hotmail.com
}

\begin{abstract}
Resumo
Acredita-se que todos os profissionais da área da saúde que lidam diretamente com exames por imagem, devam conhecer e compreender a importância da radiobiologia e radioproteção para procedimentos em que sejam necessários à utilização de raios-X, evitando assim exposições desnecessárias e efeitos nocivos das radiações ionizantes. Objetivo: Verificar o potencial dos protetores plumbíferos como método de radioproteção em consultórios odontológicos como meio de redução aos níveis de exposição à radiação ionizante. Metodologia: Constitui-se de uma revisão da literatura, realizado entre março e setembro de 2021, baseado na busca de artigos completos de revisão sistemática e matanálise dos últimos 10 (dez) anos, nos idiomas inglês e português, selecionados em consulta a PubMEd, a partir da fonte Medline, utilizando como palavras-chave: radiologia odontológica; radiação ionizante; proteção radiológica/radiobiologia; EPI’s radiológicos. Especificamente em espécie humana de estudos em espécie humana, foram filtrados 20 artigos que envolvessem doses de radiação dos 4.627 listados na busca. Considerações finais: A respeito dos conhecimentos dos Cirurgiões Dentistas, quando se estreitam e compilam as pesquisas individuais, a fim de se obter um panorama geral, é possível se estabelecer que o conhecimento sobre radiologia, e as normas de radioproteção são escassas, e por muitas vezes, a falta de conhecimento, pode levar à imperícia, e negligência, ocasionando acidentes. Os estudos demonstram de forma densa a necessidade de uma nova visão a respeito da disciplina de radiologia nos cursos de graduação, e especialização. A fim de preparar de forma adequada os futuros profissionais. Minimizando assim, possíveis acidentes inerentes a prática radiológica.
\end{abstract}

Palavras-chave: Radiologia odontológica; Radiação ionizante; Proteção radiológica; EPI radiológico.

\begin{abstract}
It is believed that all health professionals who deal directly with imaging exams should know and understand the importance of radiobiology and radioprotection for procedures in which the use of X-rays is necessary, thus avoiding unnecessary exposure and harmful effects of ionizing radiation. Objective: To verify the potential of lead protectors as a method of radioprotection in dental offices as a means of reducing levels of exposure to ionizing radiation. Methodology: It consists of a literature review, carried out between March and September 2021, based on the search for complete systematic review articles and math-analysis of the last 10 (ten) years, in English and Portuguese, selected in consultation with PubMEd, from Medline source, using as keywords: dental radiology; ionizing radiation; radiological protection/radiobiology; Radiological PPE. Specifically in humans from studies in humans, were filtered 20 articles involving radiation doses from the 4,627 listed in the search. Conclusion: Regarding the knowledge of Dental Surgeons, when individual research are narrowed and compiled in order to obtain an overall picture, it is possible to establish that knowledge about radiology and radiation protection standards are scarce, and often times, lack of knowledge, can lead to malpractice, and negligence, causing accidents. The studies strongly demonstrate the need for a new vision regarding the discipline of radiology in undergraduate and specialization courses. In order to adequately prepare future professionals. Thus, minimizing possible accidents inherent to radiological practice.
\end{abstract}

Keywords: Dental radiology; Ionizing radiation; Radiological protection; Radiological IPE. 


\section{Introdução}

O uso de exames complementares radiológicos para fins diagnósticos, agregam grande relevância na odontologia clínica, auxiliando principalmente a visualização de estruturas muitas vezes não observadas no exame clínico, facilitando assim não apenas o diagnóstico, como também a melhor indicação de tratamento e prognóstico. (Alves et al, 2016; Leite et al, 2020).

A literatura cita que em oito de novembro de 1895, Wilhelm Conrad Röntgen, descobriu o que viria se tornar a radiação, durante seus estudos com descargas elétricas em tubos, contendo conteúdos gasosos. Sem saber ao certo, que sua descoberta daria início a uma nova área de estudo e pesquisa: a radiologia, Röntgen verificou que durante o tempo de duração de descarga elétrica sobre a ampola produziu-se uma claridade, que incidia sobre uma tela fosforescente que havia disponível no laboratório. Ao repetir as descargas, Röntgen pode perceber que por mais que houvesse obstáculos entre a ampola e a tela, a luminescência, continuava sendo reproduzida. Tão logo esta energia tinha a capacidade de atravessar corpos de diferentes espessuras. (Costa et al, 2013).

Desde sua descoberta em 1895, os raios-X, ganharam espaço e aplicabilidade em várias vertentes da área da saúde, entretanto além de seus benefícios, sobretudo para qualidade dos diagnósticos; devemos nos atentar de mesmo modo aos riscos inerentes à exposição à estas radiações, sejam de forma acidental, e repetida a eles. Gerando efeitos nocivos ao tecido biológico quando ultrapassam seus limiares de dose, que podem levar a danos permanentes nas células, devido a ação e propriedade ionizante dos raios- $\mathrm{X}$, induzindo a transformações nas moléculas presentes no DNA, causando mutações, neoplasias e morte celular. Tais alterações podem ser observadas imediatamente, ou até dias, semanas, meses ou anos após, sendo chamados estes últimos de efeitos tardios da radiação. (Costa et al, 2013; Barbosa, 2020; Santana et al, 2017).

Para tal, o objetivo geral deste estudo é analisar o potencial dos protetores plumbíferos como método de radioproteção em consultórios odontológicos como meio de redução aos níveis de exposição à radiação ionizante. Bem como, orientar o profissional no uso e conservação adequados dos equipamentos e acessórios radiológicos para minimizar os riscos inerentes à radiação ionizante e propor uso de dosímetros de ambiente para controle de dose de exposição, respectivamente como objetivos específicos.

\section{Metodologia}

Constitui-se de uma revisão da literatura, realizado entre março e setembro de 2021, baseado na busca de artigos completos de revisão sistemática e matanálise dos últimos 10 (dez) anos, nos idiomas inglês e português, selecionados em consulta a PubMEd, a partir da fonte Medline, utilizando como palavras-chave: radiologia odontológica; radiação ionizante; proteção radiológica/radiobiologia; EPI’s radiológicos, especificamente em espécie humana de estudos em espécie humana, foram filtrados 20 artigos que envolvessem doses de radiação dos 4.627 listados na busca.

\section{Revisão de Literatura}

Os efeitos biológicos dos raios-X, são descritos pela literatura como estocásticos, e determinísticos. Os efeitos estocásticos são provocados por pequenas doses recebidas, durante um longo período, podendo acarretar aos pacientes patologias como catarata radiogênica, esterilidade e radiodermite. Os efeitos determinísticos são resultantes de uma alta dose de radiação ionizante, ultrapassando o limiar de segurança, em um curto espaço de tempo que é dependente do tipo de radiação e do tecido em que a radiação foi aplicada. Tem como efeito principal a morte celular, efeito este que pode levar ao inadequado funcionamento de um órgão. (Amaral et al, 2021; Jansen et al, 2020; Okuno, 2013; Oliveira, 2016).

Vistos os possíveis malefícios inerentes ao uso de radiação para fins de diagnósticos médicos ou odontológicos, em 1998, foi criada a legislação da Portaria SVS/MS n 453, de 1 de junho de 1998, que se refere aos riscos e perigos da radiação 
ionizante. Documento este que em, 20 de dezembro de 2019, foi revogado pela ANVISA, por meio da Resolução- RDC N³30, sendo um dos documentos referenciais para atendimentos radiológicos. Este novo documento aborda alterações importantes sobre pontos como: modo de uso do dosímetro, adoção de níveis de dose e definições citadas na CNEN (Comissão Nacional de Energia Nuclear), o final do Memorial Descritivo de Proteção Radiológica, RDC e Instruções Normativas, e Área controlada e área livre. Pontos antes regulados pela portaria 453, agora passam a incumbência do serviço, (definição de áreas de controle, e uso de dosímetro). (Brasil, 2019; Costa et al, 2013; Gomes et al, 2012; Jansen et al 2020; Silva 2021).

Caso todos os protocolos de biossegurança radiológicos sejam adotados de forma adequada tanto para paciente, quanto para profissional, o risco em relação a doses de radiação em radiografias odontológicas é mínimo, quando comparado com a dose natural que um ser humano pode ser exposto durante um ano, ainda neste âmbito é necessário o estudo dos efeitos de radiação tardios, que tem causalidade cumulativa de doses radiológicas. (Alves et al, 2016; Barreto, 2020; Costa et al 2017, Moura, 2020; Silva 2020).

Além das técnicas de biossegurança em radiologia, o uso de protetores plumbíferos são de grande importância para a odontologia radiológica. A blindagem contra a incidência direta dos raios-X ocorre por meio desses protetores que são constituídos por finas camadas de chumbo, que tem como papel atenuar os feixes emitidos pelos raios-X. Entre os mais relevantes para a odontologia radiológica podemos citar: os aventais, e protetores de tireoide. De acordo com os parâmetros apresentados pela RCD 330, os protetores plumbíferos individuais devem conter, espessura mínima de 0,25 mm de chumbo, e recobrir toda a extensão de tronco, área de tireoide, e gônadas durante a exposição aos raios-X. Estudos corroboram a ideia sobre a eficiência do uso de aventais plumbíferos para atenuação da radiação ionizante, visto que o protetor plumbífero individual com espessura $(0,25 \mathrm{mmPb})$ na tensão de $75 \mathrm{kV}$ foi capaz de atenuar a dose radiológica no paciente durante a exposição em até 95\%. (Gomes et al, 2012; Cirilo et al, 2021; Lima et al, 2021; Soares, 2011).

Sobre a forma correta de guardar o protetor individual de chumbo, a literatura sugere que seja estendido, pois o armazenamento, dobrado, ou amassado é inadequado poderá levar a formação de fendas na fina camada de chumbo, o que irá comprometer diretamente a atenuação dos feixes de raios-X. (Gomes et al, 2012; Oliveira et al, 2016).

Pensando-se em radioproteção do ambiente físico, é necessário citar a blindagem da sala de raios-X, que é feita por meio da cobertura das paredes com argamassa misturada a barita, (mineral de sulfato de bário), que atua como barreira de proteção, e, é fundamental para a proteção do profissional, e paciente durante a exposição aos raios-X. A barreira de proteção não tem a capacidade de impedir a transmissão, da radiação-X, ou gama, e sim de atenuá-la, e sua eficácia depende dos cálculos dosimétricos necessários para a blindagem da sala de raios-X. Para garantir a eficiência da blindagem, é feito o levantamento radiográfico da sala, e a radiometria, que se trata da medição dos níveis de radiação em áreas próximas a sala de raios-X. Os níveis devem estar dentro dos padrões exigidos pela Agência Nacional de Vigilância Sanitária (ANVISA), de acordo com a portaria federal $\mathrm{n}^{\circ} 453 / 98$, sendo realizada por profissionais capacitados. (Costa et al, 2013).

\section{Discussão}

A respeito do que a literatura nos apresenta sobre protetores plumbíferos, verificou-se que o fator de aumento de proteção, esta diretamente relacionado ao uso destes. Entretanto o fator de maior prevalência nos estudos é de fato, o conhecimento e entendimento do profissional sobre temas como radioproteção, protetores plumbíferos, e a radiologia propriamente dita. Santana et al, (2017), discute de forma ampla, o nível de conhecimento que os Cirurgiões Dentistas que realizam exames radiológicos, possuem a respeito das medidas de proteção radiológica, conclui ainda que os CD, não possuem o conhecimento necessário para uma adequada proteção radiológica durante a execução de exames radiológicos. Sobre o mesmo tema, Alves et al, (2016), corrobora a ideia, que é fundamental para a prática em radiologia odontológica, 
conhecimentos sobre biossegurança radiológica, abordando como quesito principal a proteção tanto dos profissionais como também seus pacientes. (Alves et al, 2016; Alves et al, 2021; Santana et al, 2017).

As normas de proteção radiológicas, assim como sua relação sobre os efeitos estocásticos, e determinísticas são apresentadas por Okuno, (2013), nos levando ao entendimento da real importância de tais temas não somente para a proteção do profissional e paciente, como também, para auxiliares e funcionários do local. (Okuno, 2013).

Gomes et al, (2012), relata o nível de conhecimento dos profissionais e pacientes a respeito das medidas de radioproteção. Tal estudo nos mostra a necessidade de mais informações sobre medidas de radioproteção, visto que dentre os participantes, 7 (sete), relatam já ter permanecido, como acompanhantes, sem avental de proteção plumbífero, e apenas um afirmou sempre ter utilizado avental de chumbo durante essa função. Enquanto 39\% das participantes, nunca foram questionadas sobre a possibilidade de gestação. Esses dados cooperam de forma contundente para que seja reforçado ainda durante a vida acadêmica, o ensino sobre medidas radiológicas de proteção, 43,5\% relataram que foram utilizados posicionadores para manutenção do filme na cavidade bucal. Outro fator considerado agravante para uma adequada atenuação dos raios-X, seria o armazenamento dos aventais, e protetores plumbíferos, a literatura sugere que o protetor individual de chumbo seja armazenado estendido, com intuito de preservar a fina lamínula de chumbo em seu interior, evitando assim micro fendas, que comprometem diretamente a atenuação dos feixes de raios-X. (Gomes et al, 2012).

Entretanto, Oliveira (2014), verificou que, em 71,4\% dos consultórios avaliados, o avental individual de proteção plumbífero era armazenado dobrado e acondicionado de qualquer modo, as vezes sobre o braço do equipamento, aumentado a incidência de rachaduras na lamínula de chumbo, e diminuindo a atenuação dos raios-X. E, em 5,7\% da amostra os aventais individuais eram armazenados no chão. Esse dado levanta uma importante discussão a respeito da disciplina de radiologia como parte integrante dos cursos da área da saúde, assim como também da importância da presença de um profissional treinado e habilitado para práticas radiológicas. (Oliveira, 2014).

Se faz necessário discutir a respeito das normas de radioproteção vigentes no país, e a aplicabilidade real nos consultórios odontológicos. Oliveira, e colaboradores, 2016, pautados na portaria $\mathrm{n}^{\circ}$ 453/1998, apresentam essa narrativa, por meio da análise de 43 consultórios na cidade de Patos, PB. Dos quais 94,2\% relataram não possuir sinalização de local com presença de radiação ionizante, e 85,7\% não dispõem de avisos espalhados pelos consultórios que relatem a importância do uso de aventais plumbíferos. Tais números embasam uma discussão pertinente à radioproteção, não apenas do profissional, assim como dos pacientes, e seus acompanhantes, com intuito de diminuir o risco de acidentes. Costa et al, (2013), corrobora para essa necessidade, ao explanar os riscos da exposição radiológica acidental, e sua relação com os efeitos estocásticos, e determinísticos que podem ser evitados, ou atenuados, caso as normas de radioproteção sejam colocadas em prática.

Soares (2014), aponta a necessidade do uso de protetores individuais plumbíferos para os indivíduos ocupacionalmente expostos, o que abrange não somente o Cirurgião Dentista, como seus auxiliares, estagiários, TSB, entre outros. Todavia para que as normas de proteção radiológica, sejam cumpridas de forma adequada, é necessária uma visão direcionada para a graduação dos futuros profissionais, outra alternativa são os cursos de atualização e especialização, nos quais o autor sugere que a radiologia necessita de uma educação continuada, se estendendo após o período de graduação. Tal ação se mostra imediata e relevante uma vez que já tramita o projeto de lei nº. 3.661/2012, de autoria do senador Paulo Paim, (PT/RS) que apresentou o Projeto de Lei do Senado n..$^{\circ}$ 26/2008 (PLS n..$^{\circ}$ 26/2008). Um importante projeto para a regulação da prática profissional de auxiliares, técnicos, e tecnólogos em radiologia, e especificar áreas específicas de atuação profissional. (Brasil, 2012). 
Quadro 1. Artigos selecionados.

\begin{tabular}{|c|c|c|c|}
\hline & $\begin{array}{l}\text { AUTOR } \\
\text { /TÍTULO }\end{array}$ & PARECER & COMPARATIVO \\
\hline $\begin{array}{l}\dot{0} \\
\dot{2} \\
\dot{n} \\
\dot{1} \\
\frac{1}{2}\end{array}$ & 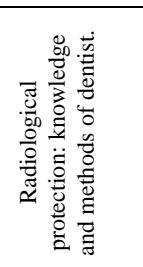 & $\begin{array}{l}\text { Este estudo contou com a participação de } 50 \\
\text { Cirurgiões Dentistas, e relata um bom nível de } \\
\text { compreensão e aplicabilidade das normas de } \\
\text { proteção em radiologia odontológica, assim como } \\
\text { interpretação dos exames. (ALVES, 2016) }\end{array}$ & $\begin{array}{l}\text { Aponta que as normas de proteção radiológica devem ser } \\
\text { respeitadas e praticadas. Relaciona as causas dos efeitos } \\
\text { estocásticos na radiologia odontológica á exposição } \\
\text { prolongada. (COSTA, 2013). }\end{array}$ \\
\hline 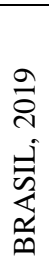 & 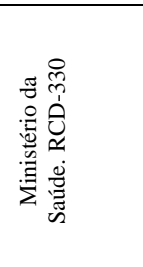 & $\begin{array}{l}\text { Apresenta a normativa do RDC- } 330 \text {. Sendo este } \\
\text { documento, um dos regentes para procedimentos } \\
\text { radiológicos. }\end{array}$ & $\begin{array}{l}\text { Baseado na portaria 453/1998, Oliveira, e colaboradores } \\
\text { avaliaram a aplicação das normas de radioproteção vigentes } \\
\text { no país em } 43 \text { consultórios de Patos, PB, e verificaram grande } \\
\text { falta de instrução, e conhecimento a respeito do tema e leis } \\
\text { regentes. (OLIVEIRA 2016). }\end{array}$ \\
\hline $\begin{array}{l}2 \\
\stackrel{n}{0} \\
\text { 䞨 } \\
0 \\
0\end{array}$ & 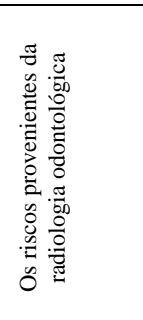 & $\begin{array}{l}\text { Reforça a necessidade de um treinamento efetivo } \\
\text { para aqueles que irão trabalhar com radiologia. } \\
\text { Ressaltando a importância, do respeito às } \\
\text { legislações, e normas vigentes em nosso país. } \\
\text { COSTA, 2013. }\end{array}$ & $\begin{array}{l}\text { O estudo aponta alguns aspectos de radioproteção em } \\
\text { desajuste com a legislação brasileira, por meio de análise em } \\
43 \text { consultórios na cidade de Patos, PB. Corroborando a ideia } \\
\text { de falta de conhecimento e aplicação dos Cirurgiões Dentistas } \\
\text { a respeito das normas de radioproteção vigentes no país. } \\
\text { Baseado na Portaria }{ }^{\circ} \text { 453/1998. OLIVEIRA, } 2016 \text {. }\end{array}$ \\
\hline 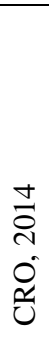 & 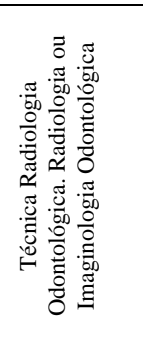 & $\begin{array}{l}\text { Apresenta de forma clara, e objetiva as diretrizes da } \\
\text { Técnica radiológica odontológica. (CRO, 2014). }\end{array}$ & $\begin{array}{l}\text { Soares discorre sobre a importância da utilização, e } \\
\text { armazenamento adequado dos protetores individuais } \\
\text { plumbíferos. (SOARES, 2014). }\end{array}$ \\
\hline 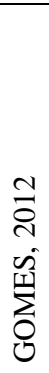 & 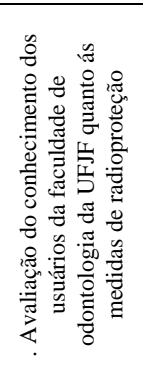 & $\begin{array}{l}\text { Relata certo nível de imperícia durante os } \\
\text { procedimentos pertinentes a radioproteção } \\
\text { odontológica, e pontua de forma abrangente o } \\
\text { conhecimento dos pacientes sobre os perigos } \\
\text { inerentes a utilização da radiação. (GOMES, 2012). }\end{array}$ & $\begin{array}{l}\text { Soares apresenta a necessidade do uso de protetor para o } \\
\text { indivíduo ocupacionalmente exposto, seja ele Cirurgião } \\
\text { Dentista, assistente, ou qualquer outro profissional que atue, } \\
\text { em ambiente com radiações ionizantes. Recomenda também, } \\
\text { instrução/educação continuada para que acidentes não } \\
\text { ocorram por motivos de negligência. } \\
\text { (SOARES, 2014). }\end{array}$ \\
\hline 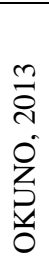 & 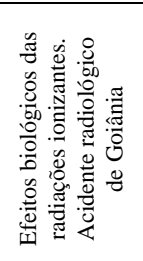 & $\begin{array}{l}\text { Relata a importância dos exames radiológicos na } \\
\text { medicina, assim como a radioterapia. Ressaltando o } \\
\text { uso dos raios-X, que deve ser sempre pautado no } \\
\text { modelo risco-benefício, de acordo com cada caso, e } \\
\text { paciente. (OKUNO, 2013). }\end{array}$ & $\begin{array}{l}\text { Aponta que as normas de proteção radiológica devem ser } \\
\text { respeitadas e praticadas. Relaciona as causas dos efeitos } \\
\text { estocásticos na radiologia odontológica á exposição } \\
\text { prolongada. (COSTA, 2013). }\end{array}$ \\
\hline
\end{tabular}




\begin{tabular}{|c|c|c|c|}
\hline 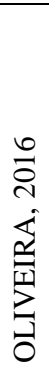 & 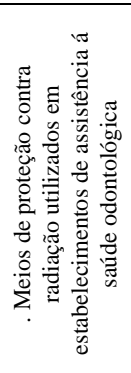 & $\begin{array}{l}\text { O estudo contou com a participação de } 43 \\
\text { consultórios odontológicos. Dos quais } 94,2 \% \text { estão } \\
\text { em desacordo com as normas de segurança da } \\
\text { vigilância sanitária. Reforçando a ideia de que o } \\
\text { cumprimento de tais normas poderiam minimizar } \\
\text { os riscos advindos da exposição á radiação. } \\
\text { (OLIVEIRA, 2016) }\end{array}$ & $\begin{array}{l}\text { O estudo apresenta casos de imperícia, e descuido mediante } \\
\text { procedimentos com radiação ionizante, assim como a falta de } \\
\text { instrução que o paciente possui sobre os perigos inerentes a } \\
\text { radiação, durante o tratamento odontológico. (GOMES, 2012) }\end{array}$ \\
\hline 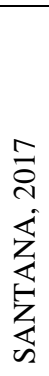 & 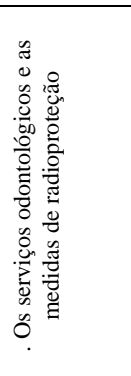 & $\begin{array}{l}\text { Conclui que a maioria dos } \mathrm{CD} \text {, que realizam } \\
\text { exames radiográficos odontológicos não possui } \\
\text { conhecimento suficiente a respeito das medidas de } \\
\text { proteção radiológica. (SANTANA, 2017) }\end{array}$ & $\begin{array}{l}\text { Este estudo contou com a participação de } 50 \text { Cirurgiões } \\
\text { Dentistas, e relata um bom nível de compreensão e } \\
\text { aplicabilidade das normas de proteção em radiologia } \\
\text { odontológica, assim como interpretação dos exames. Dado } \\
\text { que se contrapõe a ideia de que o Cirurgião Dentista possui } \\
\text { pouco conhecimento a respeito das normas de proteção } \\
\text { radiológica. (ALVES, 2016) }\end{array}$ \\
\hline 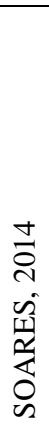 & 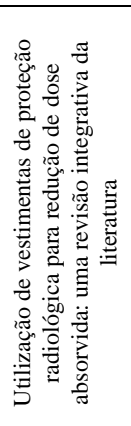 & $\begin{array}{l}\text { Soares apresenta a necessidade do uso de protetor } \\
\text { para o indivíduo ocupacionalmente exposto, seja } \\
\text { ele Cirurgião Dentista, assistente, ou qualquer outro } \\
\text { profissional que atue, em ambiente com radiações } \\
\text { ionizantes. } \\
\text { instrução/educação continuada para que acidentes } \\
\text { não ocorram por motivos de negligência. } \\
\text { (SOARES, 2014). }\end{array}$ & $\begin{array}{l}\text { Gomes e colaboradores, discorrem sobre determinado nível } \\
\text { de negligência, durante os procedimentos pertinentes a } \\
\text { radioproteção odontológica, e pontua de forma abrangente o } \\
\text { conhecimento dos pacientes sobre os perigos inerentes a } \\
\text { utilização da radiação. (GOMES, 2012). }\end{array}$ \\
\hline
\end{tabular}

Fonte: Autores.

\section{Considerações Finais}

A respeito dos conhecimentos dos Cirurgiões Dentistas, quando se estreitam e compilam as pesquisas individuais, a fim de se obter um panorama geral, é possível se estabelecer que o conhecimento sobre radiologia, e as normas de radioproteção são escassas, e por muitas vezes, a falta de conhecimento, pode levar à imperícia, e negligência, ocasionando acidentes. Os estudos demonstram de forma densa a necessidade de uma nova visão a respeito da disciplina de radiologia nos cursos de graduação, e especialização. A fim de preparar de forma adequada os futuros profissionais. Minimizando assim, possíveis acidentes inerentes a prática radiológica. Desde modo sugerimos que trabalhos futuros, quantifiquem instituições que abordam radioproteção como temática na formação dos discentes, controle de qualidade dos materiais plumbíferos utilizados em clínicas das universidades e implementação de dosímetros para profissionais para evolução da temática e aperfeiçoamento dos futuros profissionais dentistas.

\section{Referências}

Alves, W. D. A., Camelo, C. A. C., Guaré, R. D. O., Costa, C. H. M. D., \& Almeida, M. S. C. (2016). Radiological protection: knowledge and methods of dentist. Arquivos em Odontologia, 52(3), 130-135.

Alves, C. S. D. O., \& Cardoso, I. A. (2021). Desempenho dos profissionais da saúde no curso de Proteção Radiológica em um ambiente virtual de aprendizagem.

Amaral, G. S., \& Morais, Â. M. D. (2021). A importância da biossegurança na radiologia odontológica. Facit Business and Technology Journal, 1(24).

Barreto, M. S. Doses absorvidas em órgãos-alvo em TCFC e TCMD. 
Brasil. (2019). RCD-330. Ministério da Saúde.

Brasil. (2002). Projeto de lei 3661, de 10 de julho de 2002. Exercício das profissões de técnico e tecnólogo em radiologia e de bacharel em ciências radiológicas. Congresso. Senado.https://www.camara.leg.br/propostas-legislativas/540959.

Cirilo, A. N., Neto, D. P., Tessaro, G. C., Vilarim, L. T., \& de Sousa Filgueiras, P. (2021). Radiação ionizante: uma revisão de literatura.

Costa, T. G. (2013). Risks from dental radiology; Os riscos provenientes da radiologia odontologica.

CRO/SP. (2014). Câmara Técnica Radiologia Odontológica. Radiologia ou Imaginologia Odontológica.

Gomes, C. K., Duque, A. C. D. R., Dias, I. M., Martins, M. E. M. N., \& Devito, K. L. (2012). Avaliação do conhecimento dos usuários da Faculdade de odontologia da UFJF quanto às medidas de radioproteção. Odontologia Clínico-Científica (Online), 11(1), 25-30.

Jansen, L. C., \& Razuck, F. B. (2020). Os trinta anos do acidente de Goiânia e a Gestão do Conhecimento Nuclear: a atuação do Instituto de Radioproteção e Dosimetria. Brazilian Journal of Health Review, 3(6), 16108-16118.

Lima, R. S., Santos, P. V. B., dos Anjos Hora, I. A., \& de Gutierrez, G. M. (2021). Radiação ionizante na odontologia, quais os cuidados devemos ter?. Jornada Odontológica dos Acadêmicos da Católica, 6.

Moura, D. D. C., de Oliveira, G. F., \& Da Silva, F. C. A. (2020). Autoavaliação de proteção radiológica em serviços de radiodiagnóstico odontológico baseado na Portaria 453/98 ANVISA. Brazilian Journal of Radiation Sciences, 8(2).

Okuno, E. (2013). Efeitos biológicos das radiações ionizantes: acidente radiológico de Goiânia. Estudos avançados, 27, $185-200$.

Oliveira, D. H. M., Almeida, M. S. C., da Costa, C. H. M., \& de Sousa Filho, L. F. (2016). Meios de proteção contra radiação utilizados em estabelecimentos de assistência à saúde odontológica. Revista da Faculdade de Odontologia-UPF, 21(2).

Santana, A. S., Moura, J. R., \& da Silva, N. M. (2017). Os serviços odontológicos e as medidas de radioproteção. Revista da Faculdade de Odontologia$U P F, 22(2)$

Santos Leite, F., Medeiros, M. D. F. C. Z., Militão, A. O., Braga, L. G. O., \& Vasconcelos, D. S. C. Análise de conformidade de radioproteção nos serviços de odontologia radioprotection compliance analysis in dental services.

Silva, E., Ribeiro, G., Melo, J. A. C., da Silva, R. S., de Lima, S. B. S., \& de Medeiros, C. (2021). Proteção radiológica: A reflexão da ergologia sobre o trabalho em saúde. Research, Society and Development, 10(7), e56610716886-e56610716886.

Silva, L. M., Silva, G., \& Santos, W. D. S. (2020). Determinação da dose de entrada na pele devido a raio X odontológico utilizando método de Monte Carlo.

Soares, F. A. P., Pereira, A. G., \& Flôr, R. D. C. (2011). Utilização de vestimentas de proteção radiológica para redução de dose absorvida: uma revisão integrativa da literatura. Radiologia Brasileira, 44, 97-103. 